\title{
Terahertz Diffractive Optics-Smart Control over Radiation
}

\section{Agnieszka Siemion ${ }^{1}$ (D)}

Received: 15 October 2018 / Accepted: 18 March 2019 / Published online: 12 April 2019

(C) The Author(s) 2019

\begin{abstract}
Over the last 20 years, thin and lightweight optical elements have become very desirable, especially for the terahertz $(\mathrm{THz})$ range. Reduction of the volume of optical elements alongside an increase in their effective efficiency has begun a new direction of research leading to many practical applications. On top of that, diffractive optical elements can not only focus the incident beam, but also can shape the incoming wavefront into a desirable distribution or can redirect the energy. Starting from theoretical calculations of Fourier optics, diffractive elements have been transformed and nowadays form complicated structures that do not resemble a typical Fresnel lens. The precise control over a phase shift introduced by the designed element creates an opportunity to almost freely transform an incident wavefront. Moreover, the vast diversity of computer-generated holograms (also called synthetic) contributes substantially to this topic. Diffractive elements have a great impact on $\mathrm{THz}$ optical systems because their manufacturing is very simple in comparison with any other range of radiation (infrared, visible, ultraviolet, etc.). This review paper underlines developments in evolution of diffractive optics and highlights main principles and technological approaches for fabrication of diffraction optics within the terahertz range, thus serving as a guide to design and production considerations.
\end{abstract}

Keywords 040.2235 Far infrared or terahertz - 050.1970 Diffractive optics · 050.2770 Gratings · 090.1760 Computer holography · 090.1970 Diffractive optics · 090.2890 Holographic optical elements

\section{Introduction}

More than 50 years ago [1], scientists established that small thickness, low weight, and minimized absorption losses are advantageous features for optical elements. Such

Agnieszka Siemion

agnieszka@if.pw.edu.pl

1 Faculty of Physics, Warsaw University of Technology, 75 Koszykowa, Warsaw, Poland 
attributes can be achieved by using a diffractive optics approach, where the design is based not on the geometrical optics, but on the wave optics. Thus, the feature size of the modelled element is of the order of wavelength and therefore the phase shifts introduced by particular zones can be precisely controlled in order to achieve a constructive interference. This is the main advantage of diffractive elements over their refractive counterparts. Here, the phenomenon of diffraction, which sometimes may be troublesome, gives the opportunity to control the light by designing optical elements introducing particular phase shifts of the passing through radiation. Recently, $\mathrm{THz}$ beam-forming [2, 3], imaging [4], and communication [5] have started to play fundamental role in the development of $\mathrm{THz}$ technology. Thus, a precise and dedicated design of optical elements has been of such a great importance. Moreover, diffractive optics can provide even better performance in comparison to conventional lenses and enable designing elements with F-number (the ratio of focal length of the system to the diameter of its entrance pupil) lower than 1 corresponding to short focal length and large diameter at the same time [6]. The ability to replace volume elements with thin structures has become preferable at first for millimeter and then for $\mathrm{THz}$ waves. In many applications, it has been indispensable to minimize the setup assuring a large working area, a precise control over redirecting of the incident energy or shaping the beam.

Terahertz diffractive optical elements (DOEs) can be understood as structures with design governed by wave approach and thus allowing them to be smaller and lighter than corresponding refractive structures. The most popular and known diffractive elements are gratings and diffractive lenses, described in Sections 4 and 5, respectively. However, there are many more structures belonging to DOE family like diffractive lens arrays (Section 6), advanced diffractive elements forming various types of beams (Section 7), dynamically displayed diffractive elements on spatial terahertz modulators (STMs-Section 8), or holograms (Section 9). The last group allows to obtain arbitrary image at a desired plane. Such image can be larger than the hologram area, can consist of different planes and, depending on the design, can be created at different distances. Moreover, the shape of the incident wavefront can be corrected to form the truly desired image. Additional groups of elements that are thin and could be understood as kind of unconventional DOEs are sub-wave structures and metamaterials (Section 10). However, these innovative optical elements require different design methods.

Depending on the definition of the $\mathrm{THz}$ band, it can be either the radiation between $100 \mathrm{GHz}$ and $10 \mathrm{THz}$ according to $[2,7]$ or the radiation from the $300-\mathrm{GHz}$ to 10-THz range [8]. Due to the fact that millimeter and terahertz waves are closely related to each other, the MMWs can be treated as part of the THz range or as the sub-THz band, located just next to it. MMWs are defined as electromagnetic radiation with frequency ranging from 30 to $300 \mathrm{GHz}$ and they are also called extremely high frequencies EHF or Ka, $\mathrm{U}$, and $\mathrm{W}$ bands, in radio and microwave frequencies, respectively [7]. Thus, in this article, MMW band is treated as a part of the $\mathrm{THz}$ frequencies. 


\section{Designing DOEs}

Each optical beam, a wavefront, is defined by a complex amplitude $u(x, y)=$ $A(x, y) e^{i \phi(x, y)}$ describing light-field distribution that consists of two physical quantities: an amplitude $A(x, y)$ and a phase $\phi(x, y)$. The latter is crucial due to the fact that it describes the direction and character of propagation of the described wavefront. When the propagating beam illuminates particular optical element, its complex amplitude $u_{\text {in }}(x, y)$ is modified by the transmittance of this element $t(x, y)$ giving the output distribution $u_{\text {out }}$ equal to $u_{\text {in }}(x, y) t(x, y)$. In case of diffractive elements, their transmittance is determined by appropriate coding of the desired phase distribution that should be introduced by this element. Such phase delay map and type of coding result in amplitude and/or phase distributions introduced by our element. There are three parameters that determine the shape and thickness of the element: the phase distribution, the refractive index of the medium, and the design wavelength (DWL). Different methods of coding phase distribution determine the type and the efficiency of diffractive elements [9]— presented in Table 1. Each of the structures described in Table 1 has different maximal diffraction efficiency which is defined by the amplitude coefficient of the expansion of the transmittance of the grating into Fourier series [9].

Diffractive optical elements are strictly related to the design wavelength (DWL), thus making them narrowband. The phase retardation is matched due to the proper structure thickness for the particular wavelength and the refractive index of the material, introducing very large chromatic aberration. However, such a drawback can be suppressed by designing a kinoform structure of higher order [10, 11]. Kinoform of first order introduces $2 \pi$ phase shift while kinoform of higher order results in a maximal phase shift of $2 p \pi$, where $p$ is the order of a kinoform-being the natural number larger than 1. Normally, all wavelengths longer than DWL encounter too small phase retardation resulting in drastically decreased diffraction efficiency for these wavelengths, which means that such structures do not work for these wavelengths. However, in case of a high order kinoform, the DWL is related to a $p$ times

Table 1 Diffraction efficiency in relation to phase coding method for DOEs

\begin{tabular}{lll}
\hline Method of coding phase & Diffraction efficiency $\eta_{m}$ & Diffraction efficiency in 1st order \\
\hline Amplitude binary $(a=0.5)^{\mathrm{a}}$ & $\frac{\sin ^{2}\left(\frac{\pi m}{2}\right)}{(\pi m)^{2}}$ & $10.1 \%$ \\
Amplitude with different opening ratios $a$ & $\frac{\sin ^{2}\left(\frac{\pi a m}{2}\right)}{(\pi a m)^{2}}$ & Up to $10.1 \%$ for $a=0.5$ \\
Binary phase $(2-$ level $)$ & $\frac{\sin ^{2}\left(\frac{\pi m}{2}\right)}{(\pi m)^{2}}\left[1-(-1)^{m}\right]^{2}$ & $40.4 \%$ \\
Multi-level phase $(N=4,8,16 \ldots)$ & $\operatorname{sinc}^{2}\left(\frac{m}{N}\right)$ & Up to $100 \%$ \\
$N=4$ & & $81 \%$ \\
$N=8$ & & $95 \%$ \\
$N=16$ & & $99 \%$ \\
Kinoform-with continuous phase profile & $\operatorname{sinc}^{2}(1-m)$ & $100 \%$ \\
Kinoform of higher order & $\operatorname{sinc}^{2}(p-m)$ & $100 \%$ \\
\hline
\end{tabular}

${ }^{\text {a } O p e n i n g, ~ a l s o ~ c a l l e d ~ f i l l ~ f a c t o r, ~ f o r ~ b i n a r y ~ a m p l i t u d e ~ g r a t i n g ~ i s ~ e q u a l ~ t o ~} a=0.5$ 
larger phase shift; thus, all $p$ times higher harmonics are also perfectly matchedresulting in broadband working. High-order kinoform $(\mathrm{HOK})$ structures are very effective assuming that a typical detector size is of the order of wavelength or larger. Even in case of small detectors, they predominantly require larger antenna; thus, in both situations, the whole focal spot is detected.

\section{First Steps in THz Diffractive Optics}

The year 1961 was significant for THz diffractive optics-a big scientific breakthrough came when F. L. Wentworth, J. C. Wiltse, and F. Sobel developed the first diffractive element for sub-THz waves and published their results [1]. They described and manufactured Fresnel zone plates-half-period and quarter-period (that corresponds to 2- and 4-level phase structure, respectively). Then, in the 1960s, next two works describing zone plates were reported: in 1962 by M. Cohn, F. Wentworth, F. Sobel, and J. Wiltse [12] and in 1967 by G. Weibel and H. Dressel [13]. Further development took place in the 1980s, according to [14], when next research articles concerning THz diffractive optical elements (DOEs) were published in 1982 by M. Lazarus, F. Pantoja, S. Novak, and M. Somekh [15], 1983 by J. Thornton and J. Strozyk [16], 1985 by J. Wiltse [17], 1987 D. Black and J. Wiltse [18], and 1988 by B. Huder and W. Menzel [19]. These articles describe structures like Fresnel zone plates assuming the use of different phase coding methods for both reflection and transmission configurations.

First, THz diffractive elements, for frequencies higher than $0.3 \mathrm{THz}$, were reported and experimentally verified not until 2002: Fresnel zone plate lens [20], multilevel Fresnel diffractive lens [21], and THz hologram [22]. The first measurement of the diffraction grating in the time domain spectroscopy (TDS) configuration was carried out in 2005 [23]. Without a doubt, the twenty-first century has become the time of greatest and fastest developments in terahertz technique accompanied with an impressive growth in optics, especially a large group of diffractive elements.

\section{Diffraction Gratings}

The first analyzed and the most representative group of diffractive elements are gratings. They play important roles in diffractive optics, because they can be used in spectrometers, monochromators, beam redirectors, and many other optical devices. Gratings enable measuring the diffraction efficiency of elements coded by different methods (described in Table 1). According to Fourier optics, the type of phase coding of the grating specifies the amount of the energy redirected into each order of diffraction in relation to all incident energy [9] and has already been discussed in [24]. In case of DOEs' design, choosing a phase coding method is crucial not only for diffraction efficiency but also for complicity and price of the element. For further considerations, it should be underlined that simple binary diffractive lens gives information about the amount of radiation focused not only at a designed distance, but also at other shorter distances [25]. Moreover, some radiation remains unchanged and 
some is diverged. All the radiation that is not focused at the desired distance forms unwanted noise; thus, it is crucial to ensure the highest possible diffraction efficiency of the designed structure.

The simplest grating is a binary amplitude structure that blocks part of the incoming radiation, and thus achieves maximally only $10.1 \%$ of diffraction efficiency. This value decreases when a fill factor of the grating is different than 50\%. Mostly, such gratings are reported as metallic structures, very simple in manufacturing and theoretical description; thus, the focus is rather put on additional effects, like anomalous transmission of such gratings [26, 27], controlling the surface beam profile in case of circular concentric grating [28], laser beam shaping to collimate diverging radiation [3], creating vortex beams [29], arising surface plasmons, polaritons [30, 31], or prism-like behavior of metal grating with varying periods [32]. A beam can also be deflected by metamaterial structures [33].

Due to the fact that there are many transmissive materials for the $\mathrm{THz}$ radiation, additionally having a "reasonable" refractive index value (not very close to 1), not many amplitude structures have been created so far. Phase gratings have much larger diffraction efficiency. An example of binary one-dimensional Ronchi grating is shown in Fig. 1a. It can be clearly noticed that for DWL $(0.667 \mathrm{THz})$, the far field intensity distribution corresponds to the one from ideal binary phase grating - no 0th order and equal \pm 1 st order. As the frequency, and hence the wavelength, changes, the height of the structure starts to introduce phase delay different than $\pi$, the efficiency in \pm 1 st order starts to decrease, and 0th order appears (Fig. 1b). Here, it becomes clear that a proper phase shift must be assured to obtain the maximal possible diffraction efficiency, and in this case, the attenuation of the material is not taken into account. Obviously, all these considerations can be expanded to 2D gratings [35]. Factors that influence efficiency are a step height defining the introduced phase shift and the fact that grating does not have infinitesimally small thickness as it is mostly assumed [36]. In case of kinoform (blazed) gratings [37], the presence of 0th order of diffraction (and all other despite 1st) is the evidence of not perfectly matched parameters of the grating.

Blazed gratings have much larger possibilities especially that for the $\mathrm{THz}$ range, they are relatively easy to manufacture both in transmission [40] and in reflection modes [41]. Despite all advantages of kinoform gratings, there are also some drawbacks that should be taken into account while manufacturing like an angle-dependent fill factor [38] (Fig. 2a) and a shadow effect [39] (Fig. 2b), which lowers efficiency.

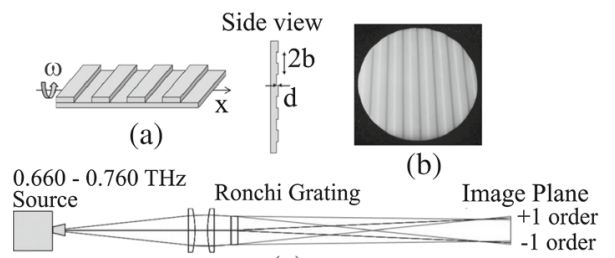

L1 L2

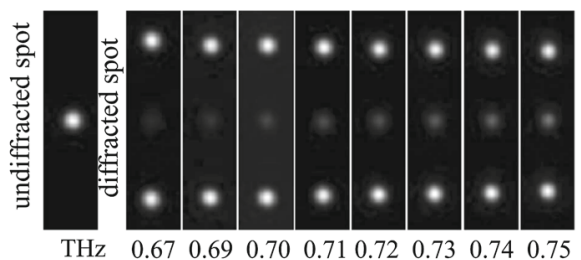

$\begin{array}{llllllllll}\mathrm{THz} & 0.67 & 0.69 & 0.70 & 0.71 & 0.72 & 0.73 & 0.74 & 0.75\end{array}$

Fig. 1 a Grating design. b Photograph. c Optical far-field configuration. (right) Measured THz spots showing the frequency-dependent evolution of the $-1,0$, and +1 diffracted orders. Reproduced from [34] 

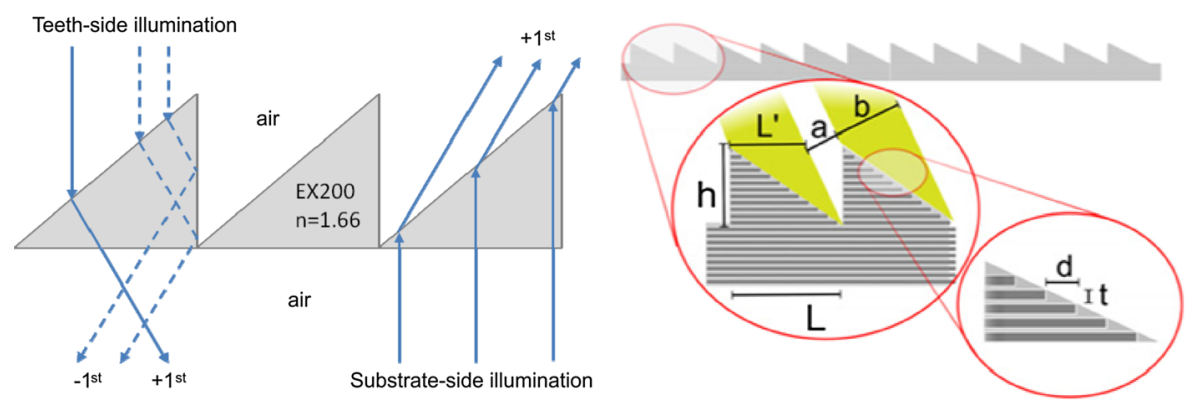

Fig. 2 Explanation of shadow effect based on geometrical optics, considering illumination from both sides (left) and at some angle (right). Dashed rays are misdirected into -1st order by the shadow effect. (right) C2016 IEEE. Reprinted, with permission, from Busch et al. [38]. (left) Reproduced from Suszek et al. [39]

Basically, all optical elements are dependent on the angle of incidence and many of them are designed only for close to on-axis functioning. On the one hand, changing an illumination angle results in a change of the effective period of illuminated grating, but, on the other hand, it also results in additional internal reflections, which redirects the radiation in the wrong way. The impact of these effects changes with varying angles - the angle indicating the relief of the grating and the incidence angle-but can be suppressed by designing double-sided structures (thus, the relief becomes two times smaller) or illuminating the structure from the flat side of the substrate [42]. A shadow effect is also very dependent on the relief height which is related in the particular case to the refractive index of material. Thus, increasing the refractive index decreases the shadow effect due to the smaller step height. However, in such a case, the reflection from the surface (Fresnel losses) is larger.

In the $\mathrm{THz}$ range of radiation diffraction, gratings are also used as beam splitters $[43,44]$. They have been applied to couple the light into a waveguide with the silicone grating engraved at its top [23]. Even an electrically controlled grating device with a nematic liquid crystal (NLC) has been reported [44].

\section{Fresnel Zone Plates and Diffractive Lenses}

Due to the nonconsistent naming of different types of lenses in the literature, this section gives some definitions for simplicity. There is no doubt what refractive lens is (called also simple lens or volume lens) (Fig. 3a). Then, a thinner lens consisting of multiple parts (used for the visible light in lighthouses from 18th century) is called a Fresnel lens (Fig. 3b). These two types of lenses are designed using geometrical optics and the size of each "zone" has thousands of wavelengths. A totally different design process is carried out in case of diffractive optics-based on wave design where structure details are of the order of magnitude of the design wavelength. Here, structures designed using different methods of coding of phase delay map can be distinguished creating amplitude or phase elements. Amplitude structures (Fig. 3c) block part of the radiation and have lowest diffraction efficiency. However, phase 
a)

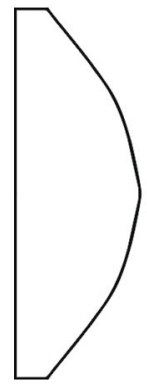

b)

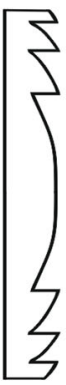

Refractive optics c)

d)

e)

f)

g)

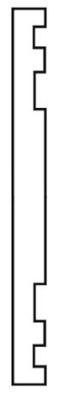

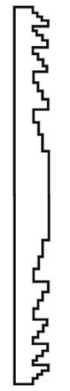

\section{Diffractive optics}

Fig. 3 Refractive (a, b) and diffractive (c-g) lenses. a Bulk lens. b Fresnel lens (not used in the THz range of radiation). c Fresnel zone plate-amplitude binary. d Planar dielectric zone plate-structure created from two materials with different refractive indices that corresponds to phase binary grating. e Binary phase_phase-reversing zone plate. f Multi-level zone plate. g Kinoform (Fresnel diffractive lens). Defined in Table 1. Adapted on the basis of [45]

structures can have different profiles-binary, multi-level, or kinoform-illustrated in Fig. 3d-g and defined in Table 1. Binary phase elements can consist of either two materials of the same thickness or one material having different thicknesses, thus introducing two different phase shifts. The difference between using refractive and diffractive lens is illustrated in Fig. 4-the performance is similar, but the volume of the material and the thickness are incomparable.

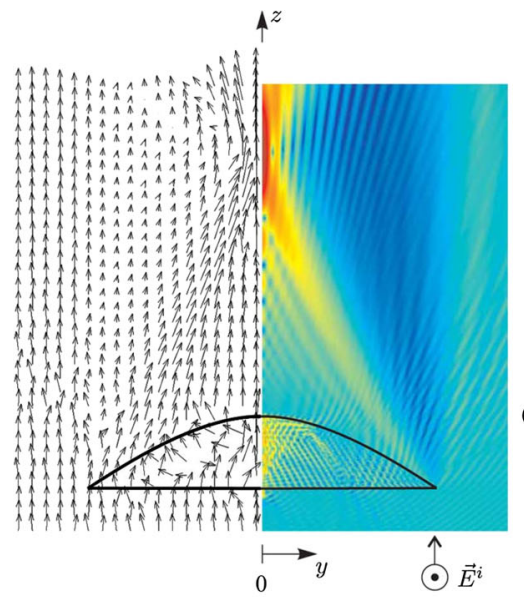

(a)

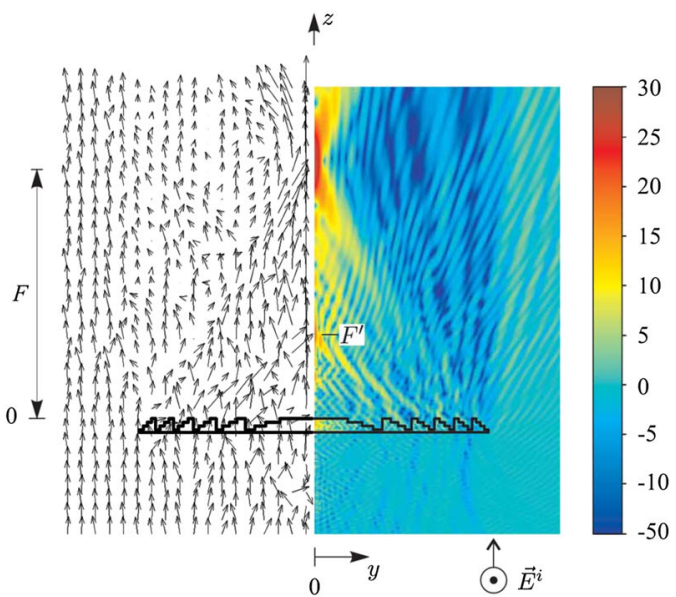

(b)

Fig. 4 Magnitude and direction of the time average of the Poynting vector-comparison of hyperbolic lens (a) with phase correcting zone plate (b), having the same focal lengths and diameters. (C) 2007 IEEE. Reprinted, with permission, from Reid et al. [46] 
Due to extensive applications, thin lens-like structures have been designed and manufactured by many groups for almost 60 years. The first research concerning $\mathrm{THz}$ and sub-THz diffractive optics was related to James C. Wiltse-the research team carried out numerous investigations of Fresnel zone plates (FZPs) [1, 14, 17, 18, 45, 47-50]. This research analyzed the shape of FZPs and invented tilted cuts of each groove-so called stepped conical zone plate. Efficiency and operating angles were also discussed. Additionally, feed considerations, losses, far-field patterns, multiplefrequency operation, and many more were analyzed. A thorough description of Fresnel zone plate antennas (FZPA) in different configurations was given by groups of Matti H. A. J. Herben and Hristo D. Hristov- [51-57]. Their work on FZPAs was supplemented by Paul F. Goldsmith [58] and Glenn S. Smith groups [46, 59] and [60]. At some point, a need to develop technology for efficient manufacturing of FZPs arose, and thus David R. S. Cumming and Edward D. Walsby groups contributed with [20, 21, 61-64], together with group of Boris A. Knyazev [65-69]. The manufacturing of multi-step lenses is a demanding task and in many cases requires etching or laser ablation processes as shown in Fig. 5. The last group continued research in applying the diffractive optics for imaging purposes with free electron laser illumination [70] and [71]. The group of Gintaras Valušis described manufacturing of lenses by laser patterning of silicon [72], next used for imaging [73], or used laser ablation to integrate FZP on-chip of detector [74]. They also proposed a manufacturing technique using cross-shaped apertures (being a resonant filter) [75-78] to select the $\mathrm{THz}$ frequencies (Fig. 6) and here, a thin structure with focal length smaller than the lens diameter was designed which unequivocally determined dominance of DOEs over their refractive counterparts in such applications. Using smaller $\mathrm{THz}$ frequencies (in the range of $0.3 \mathrm{THz}$ ) opened a new possibility for optics because a feature size could be larger and thus also other materials could be used [79, 80].

Other papers state that the distribution corresponding to Fresnel zone plate can be obtained by illuminating the silicon wafer by either imaging on it a mask [81] or using a DLP projector $[82,83]$. The latter method allows for displaying dynamically reconfigurable distribution in the FZP plane.

FZPs can also be used in a more eccentric manner-THz FZP (copper plate) as tunable THz filter [84] or IR FZP can act as THz antenna [85].

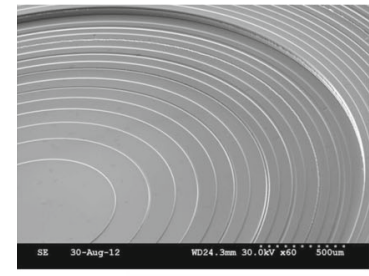

a)

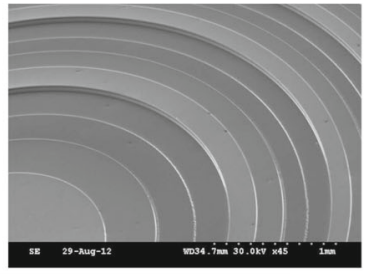

b)

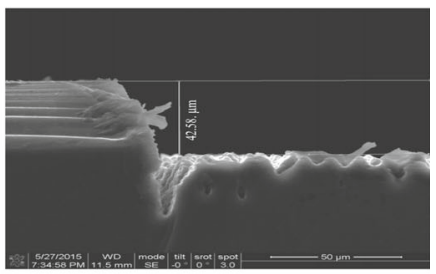

C)

Fig. 5 SEM images tilted at $60 \mathrm{deg}$ of $\mathbf{a}$ 16-level and b 4-level lenses and c cleaved lens fragment. (C) 2013 IEEE. Reprinted, with permission, from Saha et al. [64]. Reproduced from Komlenok et al. [68] 

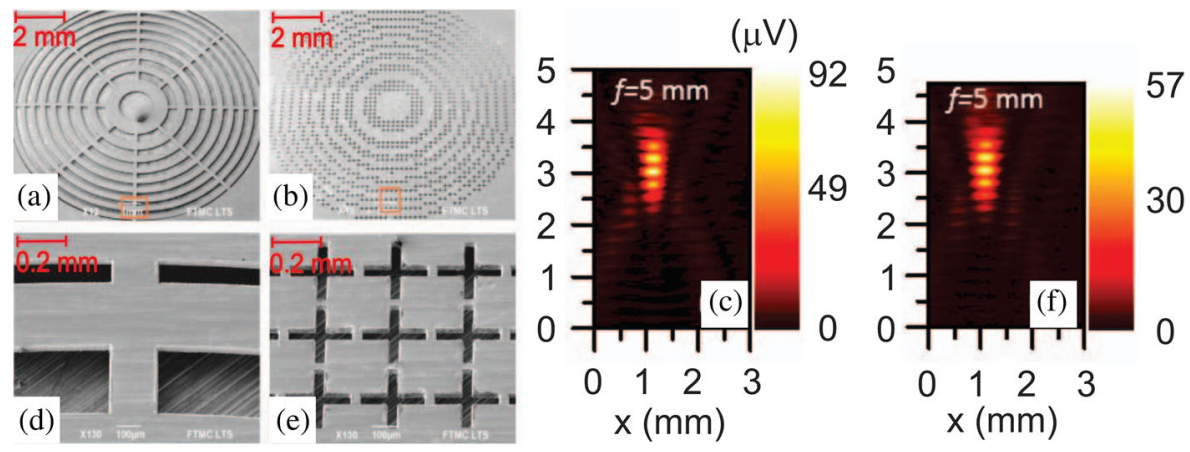

Fig. 6 The SEM images of the diffractive optical components with the focal length of $5 \mathrm{~mm}$ and the diameter of $16.5 \mathrm{~mm}$. a The Fresnel zone plate and $\mathrm{b}$ the terahertz zone plate (TZP) both processed on metal film. d, e correspond to magnified regions marked in a and $\mathbf{b}$ with red rectangles. Images of the $\mathrm{THz}$ beam focused with the conventional zone plate (c) and the TZP (f). Pixel size $25 \times 25 \mu \mathrm{m}^{2}$. (C) 2015 IEEE. Reprinted, with permission, from Minkevičius et al. [77]

\section{Lens Arrays}

Generally, lenses have two main properties-focusing the radiation and ability to form images. Indeed, these features become crucial in case of coupling the light into a detector to increase the amount of incident radiation or to perform light-field (plenoptic) imaging. Efficient functioning of the matrix of lenses was theoretically discussed in [86] by comparing a seven-step diffractive small lens array with an extended hemispherical lens. Described refractive and diffractive approaches had similar efficiency, but the latter counterpart should have had the limited working bandwidth. A diffractive design was used to focus the radiation on the matrix of detectors in [87] and [88] to improve the coupling efficiency and decrease the optical cross-talk. Lens arrays can be also designed as metasurface flat lens array [89].

\section{Advanced Diffractive Optical Elements}

The burst of diffractive optical elements having a different design idea than typical grating or Fresnel diffractive lens for the THz radiation started in 2010; however, first $\mathrm{THz}$ theoretical works go back to early the 2000s [90] and [91]. Obviously, the first sub-THz optical element was reported even earlier-in the 1990s and was able to generate Laguerre-Gaussian modes using a spiral phase plate [92]. Therefore, the design of more advanced optical elements to match a particular application has become very interesting and will be discussed in this section.

More advanced structures can be formed on the basis of a simple Fresnel diffractive lens that can serve as a part of a more complicated system. Such an element can focus and bend the radiation to be outside its geometrical shadow [93]. Thus, a peripheral area of the distribution corresponding to the lens is used as an optical element, designed using an off-axis approach. 
To obtain better performance of focusing, the typical design of the Fresnel zone plate can be substituted by fractal and Fibonacci lens-like structures [94]. The Fibonacci structure has been used for bifocal imaging which enables obtaining better resolution [95]. Additionally, DOEs can focus the incident wavefront into different shapes of focal curves [96, 97]-like segments shorter and longer than the size of the structure, rings, lines, and matrix of points [98, 99]. Designing process can contain correction of incident wavefront influence, which mostly is not a plane wave. Then, it is necessary to determine parameters of illuminating beam consistent with experimental conditions. Knowing them, during simulation, the performance of the designed structure can be verified, assuming real illumination conditions, and can be corrected according to needs [24]. Sometimes, it is even necessary to introduce a correcting layer helping to obtain larger diffraction efficiency due to suppressing the influence of the material thickness even by a thin DOE [100].

Another type of diffractive structures of a particular interest constitutes elements that generate focal distribution extended along optical axis, also called elements with increased depth of focus. In the same group, we can consider elements that form non-diffractive vortex beams. Such DOE performance can be obtained using different approaches, for example, by using two diffractive structures - one resembling a "drop" and second like a typical lens (Fig. 7c). Such two structures are able to create

(a)

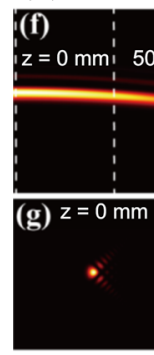

(b)

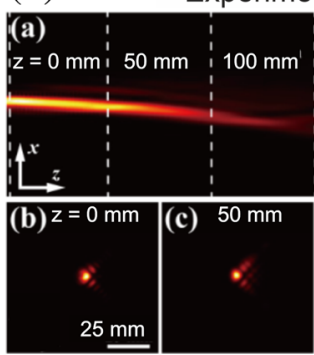

Simulation results

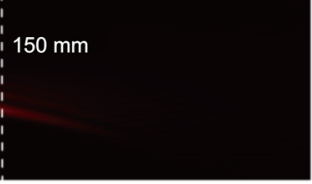

(c)

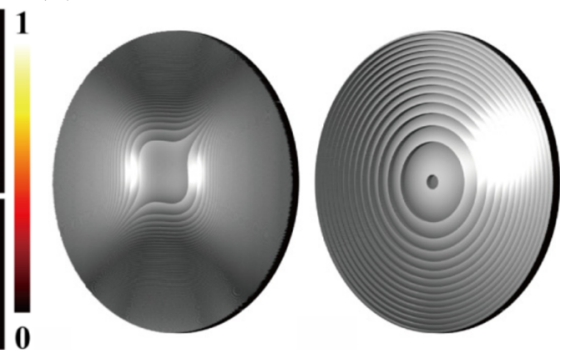

(d)

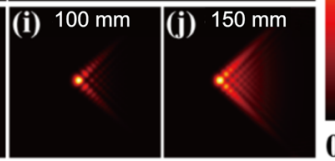

(a)

Fig. 7 Simulation (a) and experimental (b) results of the finite-energy accelerating THz airy beam. The upper row indicates the $x z$-normalized intensity profiles of the airy beam. The lower row indicates the $x y$ normalized intensity profiles of the experimentally generated airy beam measured at the dash line positions of the upper image. c Profiles of two elements creating airy beam. Additionally, experimental results of airy beam creation for "damaged" lens are shown (d). Reproduced from [101] 
airy beam with diffraction-free distance reported to be equal to $100 \mathrm{~mm}$ [101]. Moreover, if a part of the diffractive element has been covered by metal foil, a very similar diffractive pattern has been observed, and thus the authors report "healing" property of such distribution (Fig. 7d).

However, typically, a non-diffractive Bessel beam is generated by the use of axicon-like structures [102] or plate with spiral phase distribution [103]. An axicon is an element having the shape of a cone that focuses the radiation into a line segment along optical axis. Instead of a bulk structure, a diffractive alternative can be used [104] assuring the same intensity and shape of the transformed wavefront (Fig. 8). The comparison of Bessel beam generation by an axicon, a helical axicon and a spiral phase plate [105, 106], gives the alternative to use thinner structures to obtain vortex beams. Spiral binary structures manufactured in silicone [103, 107] can be sucessfully used to form non-diffractive Bessel beams. Also, more complicated configurations were used as a spiral phase plate with two 3D-printed DOEs correcting structures [108], a complementary V-shaped antenna structure [109], being a kind of metamaterial, or using photo-generated carriers displaying a computer-generated hologram forming a $\mathrm{THz}$ vortex beam [29]. Complex patterns can be displayed by spatial terahertz modulators (STMs) described in Section 8. It is a convenient way to form airy [110] or vortex beams [111].

Also, a spiral-like shape is used to create a gradient index lens (GRIN lens) focusing the radiation into a diffraction-limited spot [112]. The effective refractive index is introduced by changing spacing between following polystyrene and air slabs and acts like a lens despite its polarization.

Normally, diffractive optical elements have huge chromatic aberrations which can be partially suppressed by the use of different methods of coding phase-called the high-order kinoform, described in Section 2. Introducing a particular phase shift in a DOE is strongly dependent on the wavelength; thus, design wavelength (DWL) is a crucial parameter. The possibility of using DWL and its higher harmonics to eliminate chromatic aberration was discussed in [10] and [11].
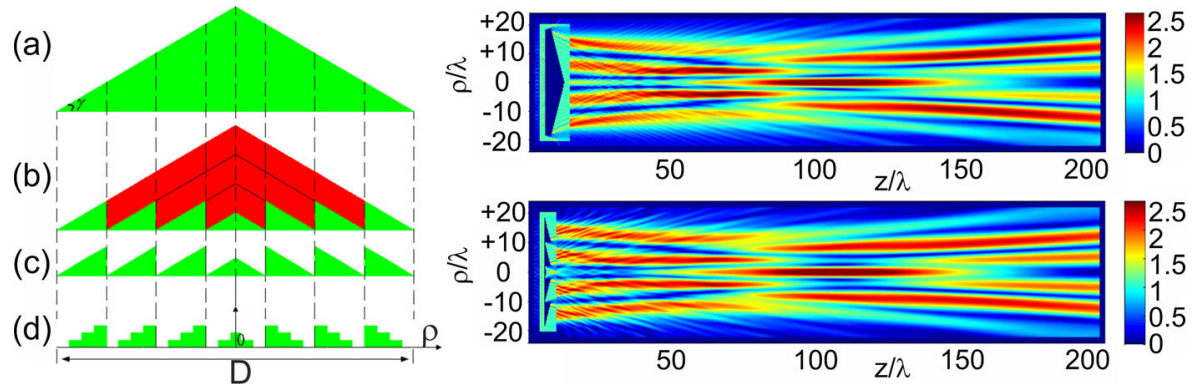

Fig. 8 (left) The design process of a diffractive axicon. a A bulk axicon. b An axicon with removed unwanted material (red part). c An equivalent kinoform axicon. d An equivalent 4-level phase axicon. (right) Electric-field amplitude patterns plotted in a pseudo-color representation: (upper) for the bulk axicon, (lower) for designed axicon (d). Reproduced from [104] 


\section{Spatial Terahertz Modulators (STMs-THz SLMs)}

This section is devoted to a dynamic displaying of the desired light-field distribution corresponding to the functioning of spatial light modulator for the visible light. Devices forming amplitude or phase distributions at the particular plane have been given for the $\mathrm{THz}$ frequencies. In this plane, a semiconductor $\mathrm{Si}$ wafer is mounted and on its surface, a pattern of photo-generated carriers is created by illumination with control beam $[111,113]$. The density of these carriers determines the transmission of $\mathrm{THz}$ beam. The pattern on the wafer has been displayed using conventional SLM, due to the fact that 800-nm-fs laser has been used. Additionally, graphene-based STMs have been reported $[114,115]$. STMs can be also designed with C-shaped metasurface [110]. Using active terahertz metamaterial also enables designing a modulator of $\mathrm{THz}$ waves controlled by external voltage [116].

(a) Incident
beam intensity
in $\left(\mathrm{X}_{0}, \mathrm{Y}_{0}\right)$-plane

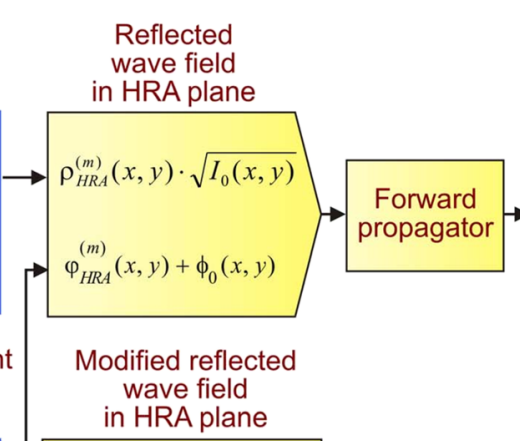

Wave field
in focal plane

Resulting in focal plane in focal plane

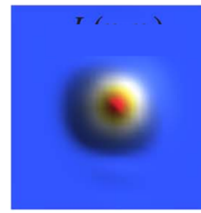

$\rightarrow$

Retrieved incident beam intensity in $\left(\mathrm{X}_{0}, \mathrm{Y}_{0}\right)$-plane in HRA plane
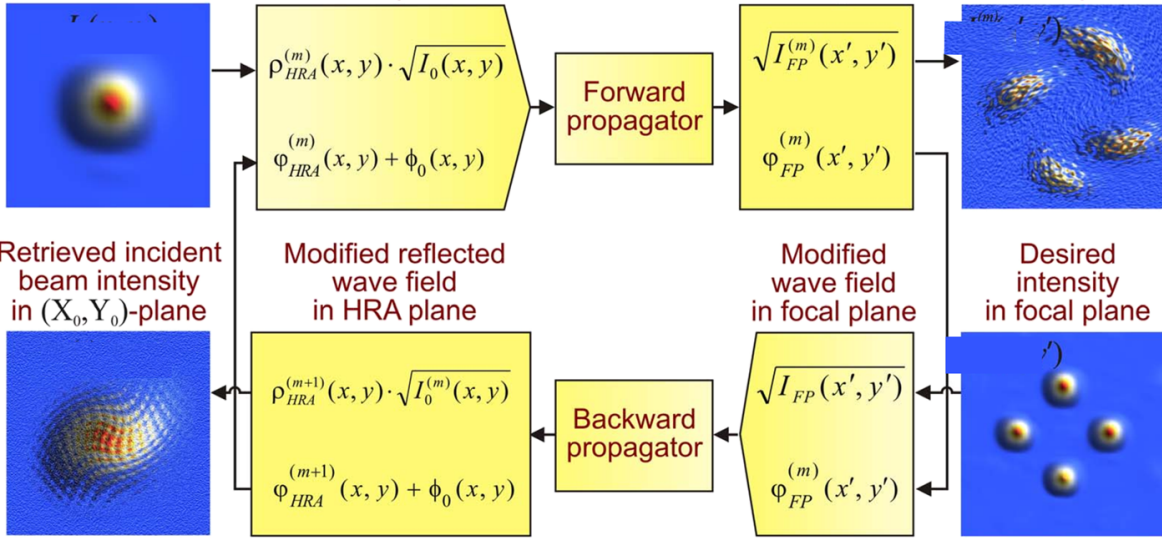

\section{Desired}

Modified wave field in focal plane

intensity in focal plane

(b)

(c)
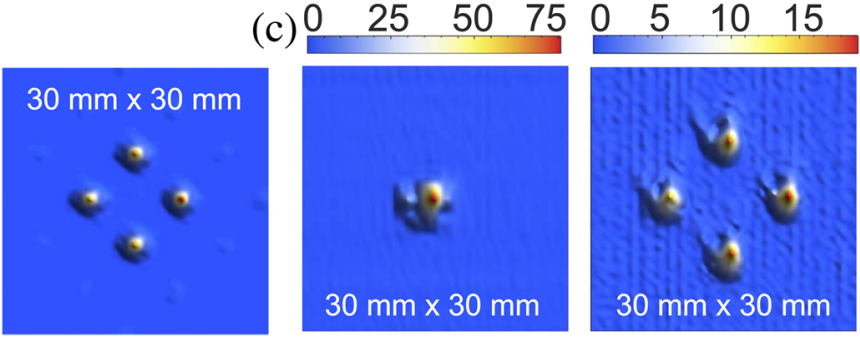

Fig. 9 a The work-flow of the Gerchberg-Saxton iterative algorithm for retrieving surface distribution. b, $\mathbf{c}$ Focusing performance of the holographic metasurface. Theoretical (b) and experimentally measured (c) intensity distributions in the focal plane at the frequency of $0.35 \mathrm{THz}$ forming one and four focal spots, respectively. Reproduced from [117] under Creative Commons Attribution 4.0 International License (https://creativecommons.org/licenses/by/4.0/) — Fig. 2 from original article has shortened caption, parts of Fig. 6 are used with rephrased caption 


\section{THz Holograms}

Computer-generated holograms (CGHs), also called synthetic, are a kind of very advanced DOEs. Mostly, the design of CGH requires using some additional methods increasing their efficiency-like the Gerchberg-Saxton algorithm [118]— (Fig. 9), which is also the case in described examples. In each step of iterating phase distribution, the algorithm forces desired amplitude distribution in the hologram plane and in the image plane. After the first iteration, the obtained image is not satisfactory, but after repeating this process few times, the quality of the reconstructed image increases significantly. Moreover, due to computer design, an arbitrary image can be created.

CGHs have been used to generate vector beams and enabled dynamic regulation of displayed distribution [29]. Synthetic holograms can be used as competitive modelling of Bessel and vortex beams in the $\mathrm{THz}$ region [22]. The conventional design method is described in Section 7. Terahertz holograms have been also used to generate a matrix of spots using the Gerchberg-Saxton algorithm [63, 117]. Such an element corresponds to the performance of the Dammann grating realizing fan-out pattern [98] and may become very useful for MIMO (multiple-input and multiple-output) technology.

Available manufacturing methods for the $\mathrm{THz}$ radiation open up many possibilities to construct dedicated phase plates like synthetic holograms able to create
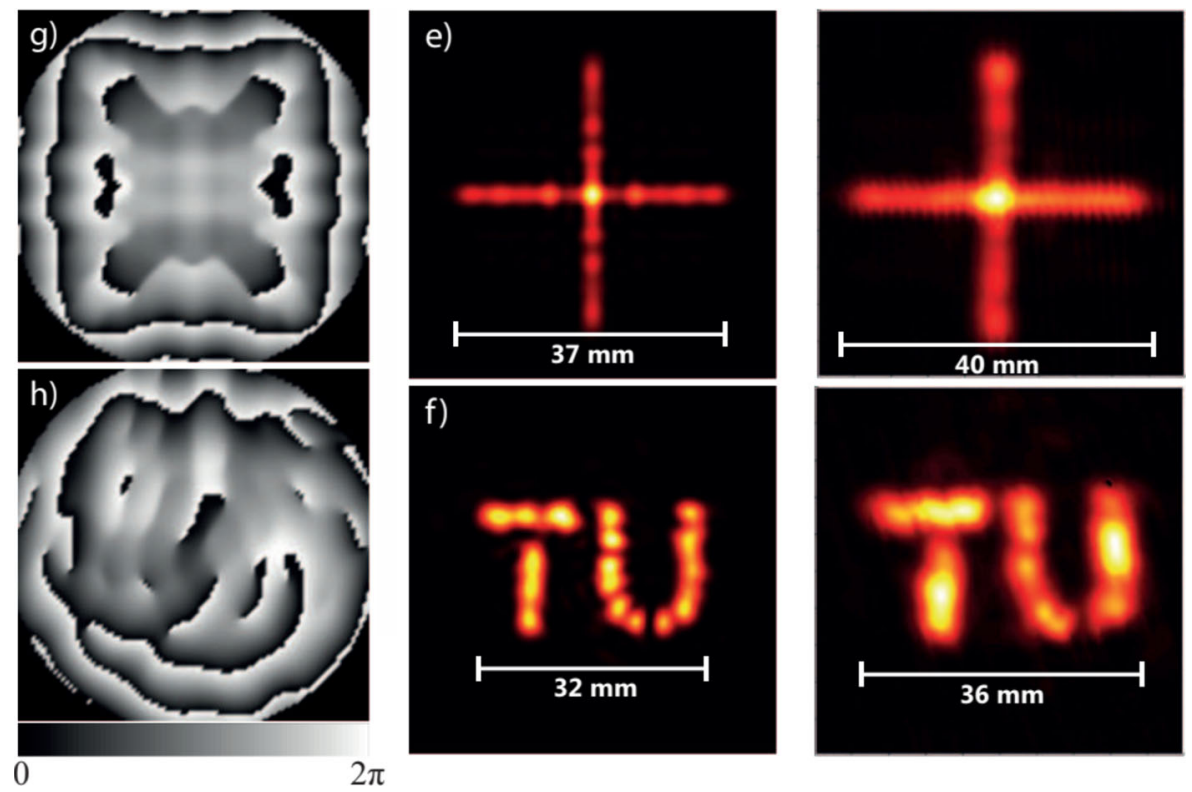

Fig. 10 Calculated phase modulation profiles (left). Calculated intensity distributions (middle) after the last iteration step of the GS algorithm. Experimental intensity distributions (right) of the optical field produced by an incident $\mathrm{THz}$ beam that was phase modulated by the two printed elements. Reproduced from: Gospodaric, J., Kuzmenko, A., Pimenov, A., Huber, C., Suess, D., Rotter, S., Pimenov, A.: 3dprinted phase waveplates for THz beam shaping. Applied Physics Letters 112(22), 221104 (2018), https:// doi.org/10.1063/1.5027179, gospodaric20183d, with the permission of AIP Publishing and author 
almost any desired distribution at a particular plane behind the hologram [119]. Two holograms—one generating "TU" letters and second a cross-are illustrated in Fig. 10.

\section{Sub-wavelength Structures and Metamaterials}

In this review article, different diffractive optical elements are discussed. These thin and lightweight components are subject to the phenomenon of diffraction. Planar structures may also be obtained by using subwave design or metamaterial structures. Mostly, they can be modelled using the effective grating theory, finite-difference time-domain (FDTD) method, effective medium approximation, or amplitude and phase retrieval algorithms. Here, they are mentioned to introduce the possibility of obtaining flat elements resembling DOEs rather than bulky refractive optics.

Even a simple metallic grid has been reported to introduce an effective refractive index profile and act as a subwave structure [32], which has also been considered in case of surface waves [120]. Sub-wavelength metal slits with additional depthmodulated grooves can redirect the energy along some angle (forming directional beams) [121]. Off-axis regime with effective grating theory enabled sub-wavelength design of a diffractive lens based on a resonance domain grating theory [122]. During designing subwave structures, principles of photonic crystal devices can be applied to create a lens [123]; however, no experimental verification has been carried out. Moreover, thin metal subwave gratings can work as polarizers [124] or triangular surface-relief sub-wavelength gratings can decrease the reflection forming AR (antireflection) coating [125-127]. In these cases, the feature of the designed element

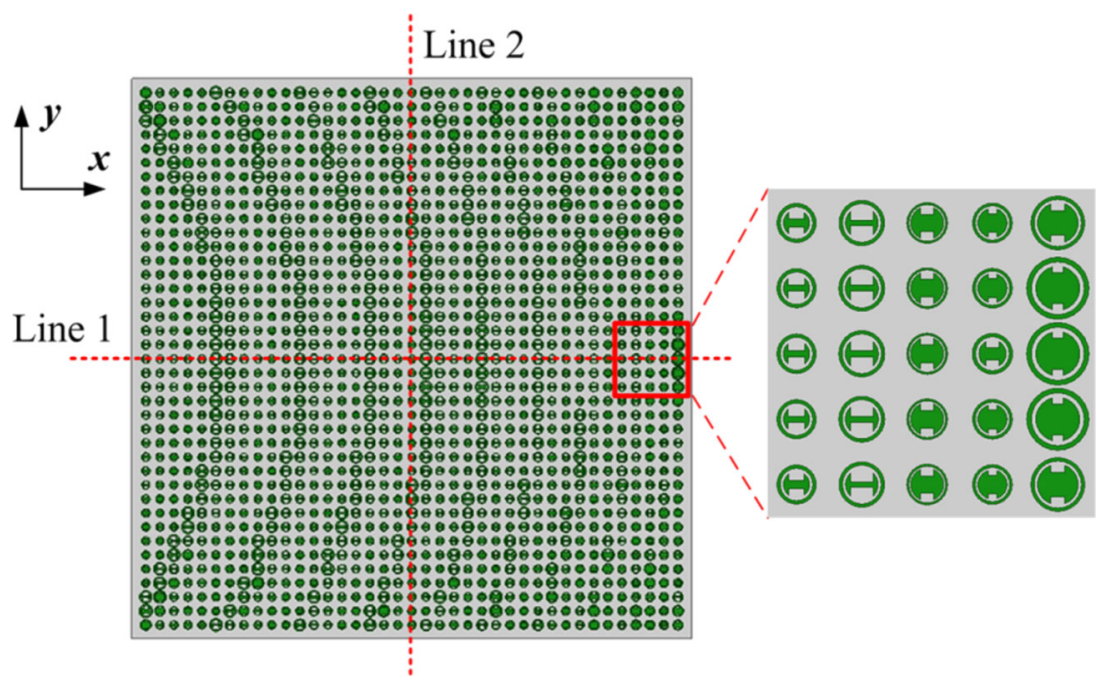

Fig. 11 Element arrangement of the THz transmitting and reflective metasurface. Reproduced from [136] under Creative Commons Attribution 4.0 International License (https://creativecommons.org/licenses/by/ $4.0 /$ ) — part a of the whole original figure 
is smaller or similar to the wavelength; thus, some effective refractive index profile is introduced, while the metamaterial structures generate abrupt phase changes [33, 128]. The subwave design has also been used to create hybrid lenses to increase their performance and reduce their volume [129, 130].

Already-described hologram structures generated by morphing patches to splitring resonator elements [117] have enabled creating a $2 \times 2$ matrix of points from one source beam (Fig. 9). The C-shaped metamaterial design allows to obtain the rectangular FZP profile, wave-deflecting structure [33] and flat lens array [89], to create 1 to 4 (multifocus) beams (with the result corresponding to Dammann structure) [131] and to create the spatial terahertz modulator (STM) which displayed four-focus lens [110]. Another type of V-shaped metamaterials has been reported to form vortex beams [109], planar lenses [132], and thin lenses with long focal depth [128]. Flat lenses have been also designed with the use of square-like metamaterial [133] or tri-layer metasurface [134, 135]. The metamaterial structure (circular shape metastructure with H-shaped elements) is used for phase compensation, thus can be treated still as diffractive [136](Fig. 11). Metasurface with circular slits can form vector beam creating vortex [137].

\section{Conclusions}

Bearing in mind that the last 20 years has brought tremendous evolution in the field of THz diffractive optics, it should be emphasized that designing thin and lightweight THz optics is still one of the most crucial goals to complement growing research achievements concerning efficient $\mathrm{THz}$ emitters and detectors also working in room temperatures. Some of the possible development paths are presented in the form concluding remarks.

Diffractive optical elements for the $\mathrm{THz}$ range of radiation are unique in a way that their manufacturing is much more accessible and simple in comparison to any other part of radiation. Nowadays, techniques of 3D printing, cutting, milling, ablating, or etching easily allow to create such elements with appropriate resolution and transmittance; thus, many advanced shapes and ideas may be executed. The easiness in manufacturing diffractive optical elements for this range of radiation also may open a new way in designing "flat" diffractive elements on not-flat surfaces, thus combining refractive and diffractive optics in an easy way.

Using new materials gives also the opportunity to focus effectively the radiation on detectors - using single lenses or arrays, which opens up the door to efficient detection.

It should be underlined that using kinoform structures of higher order diffractive elements can be designed not only for narrowband but also for broadband functioning, at the same time suppressing chromatic and geometric aberrations. Using an iterative optimized design to redistribute or shape the incoming radiation in a desired way is a very promising direction. Here, it should be also emphasized that diffractive optics gives the possibility to obtain the desired distribution larger than the size of a diffractive element in a relatively easy way, which allows for the miniaturization of the system maintaining large active area. Moreover, in case of focusing elements, such a design allows to obtain values of $F$-number below 1 . 
Taking into account all possible applications of THz waves [138], it becomes more and more crucial to fully control the beam and to be able to display different shapes at particular distances. Assuming practical utilization of the $\mathrm{THz}$ radiation, it should be obvious to incorporate $\mathrm{THz}$ diffractive optics in such fields as non-destructive industrial testing [139-141], security [142-144], and automotive industry [145-147]. Moreover, the diffractive wave approach could be expanded with the help of subwave structures or metamaterials, which seems to be future continuation of basic optical designing.

In all considerations described in this article, the polarization has not been discussed due to the fact that in dielectric materials, it has relatively insignificant influence, but in case of metal structures, meshes, or metamaterials, it is a subject for further considerations.

$\mathrm{THz}$ diffractive elements open up a new way of designing optical setups for the $\mathrm{THz}$ range - they enable creating dedicated solutions to meet the requirements of radiation, joining the world of optics and electronics.

Acknowledgements The author would like to express sincere thanks to Prof. Maciej Sypek and Prof. Andrzej Kolodziejczyk for helpful discussions and suggestions. The author gratefully acknowledges Prof. Gintaras Valušis for his kind assistance. The author is greatly indebted to Dr. Norbert Palka for all his support.

Open Access This article is distributed under the terms of the Creative Commons Attribution 4.0 International License (http://creativecommons.org/licenses/by/4.0/), which permits unrestricted use, distribution, and reproduction in any medium, provided you give appropriate credit to the original author(s) and the source, provide a link to the Creative Commons license, and indicate if changes were made.

\section{References}

1. FL Wentworth, JC Wiltse, and F Sobel. Quasi-optical surface waveguide and other components for the 100-to 300-gc region. IRE Transactions on Microwave Theory and Techniques, 9(6):512-518, 1961.

2. Daniel Headland, Yasuaki Monnai, Derek Abbott, Christophe Fumeaux, and Withawat Withayachumnankul. Tutorial: Terahertz beamforming, from concepts to realizations. APL Photonics, 3(5):051101, 2018.

3. Nanfang Yu, Romain Blanchard, Jonathan Fan, Qi Jie Wang, Christian Pflügl, Laurent Diehl, Tadataka Edamura, Shinichi Furuta, Masamichi Yamanishi, Hirofumi Kan, et al. Plasmonics for laser beam shaping. IEEE Transactions on Nanotechnology, 9(1):11-29, 2010.

4. Daniel M Mittleman. Twenty years of terahertz imaging. Optics express, 26(8):9417-9431, 2018.

5. Thomas Kleine-Ostmann and Tadao Nagatsuma. A review on terahertz communications research. Journal of Infrared, Millimeter, and Terahertz Waves, 32(2):143-171, 2011.

6. James C Wiltse. Diffraction optics for terahertz waves. In Terahertz for Military and Security Applications II, volume 5411, pages 127-136. International Society for Optics and Photonics, 2004.

7. R Boquet, N Breuil, L Chusseau, J-L Coutaz, P Crozat, J Demaison, L Duvillaret, G Gallot, F Garet, J-F Lampin, et al. Optoélectronique térahertz, 2008.

8. Xi-Cheng Zhang and Jingzhou Xu. Introduction to THz wave photonics, volume 29. Springer, 2010.

9. Joseph Goodman. Introduction to fourier optics. 2008.

10. JR Middendorf, DA LeMaster, M Zarepoor, and ER Brown. Design of multi-order diffractive THz lenses. In Infrared, Millimeter, and Terahertz. Waves (IRMMW-THz), 2012 37th International Conference on, pages 1-2. IEEE, 2012. 
11. J Suszek, AM Siemion, N Błocki, M Makowski, A Czerwiński, J Bomba, A Kowalczyk, I Ducin, K Kakarenko, N Pałka, et al. High order kinoforms as a broadband achromatic diffractive optics for terahertz beams. Optics Express, 22(3):3137-3144, 2014.

12. M Cohn, F Wentworth, F Sobel, and J Wiltse. Radiometer instrumentation for the 1 to 2 millimeter wavelength region. Proc IRE, pages 537-541, 1962.

13. Gerhard E Weibel and Herman O Dressel. Propagation studies in millimeter-wave link systems. Proceedings of the IEEE, 55(4):497-513, 1967.

14. James E Garrett and James C Wiltse. Fresnel zone plate antennas at millimeter wavelengths. International Journal of Infrared and Millimeter Waves, 12(3):195-220, 1991.

15. MJ Lazarus, FR Pantoja, S Novak, and MG Somekh. Sensitivity to direction of motion of a self-oscillating-mixer doppler radar. In IEE Proceedings F-Communications, Radar and Signal Processing, volume 129, pages 233-240. IET, 1982.

16. JW Thornton and JW Strozyk. Mcpr-an lpi wideband cable replacement radio. In SOUTHCON'83, 1983.

17. James C Wiltse. The fresnel zone-plate lens. In Millimeter wave technology III, volume 544, pages 41-48. International Society for Optics and Photonics, 1985.

18. Donald N Black and James C Wiltse. Millimeter-wave characteristics of phase-correcting fresnel zone plates. IEEE Transactions on Microwave Theory and Techniques, 35(12):1122-1129, 1987.

19. B Huder and W Menzel. Flat printed reflector antenna for mm-wave applications. electronics Letters, 24(6):318-319, 1988.

20. S Wang, T Yuan, ED Walsby, RJ Blaikie, SM Durbin, DRS Cumming, J Xu, and X-C Zhang. Characterization of t-ray binary lenses. Optics letters, 27(13):1183-1185, 2002.

21. ED Walsby, S Wang, J Xu, T Yuan, R Blaikie, SM Durbin, X-C Zhang, and DRS Cumming. Multilevel silicon diffractive optics for terahertz waves. Journal of Vacuum Science \& Technology B: Microelectronics and Nanometer Structures Processing, Measurement, and Phenomena, 20(6):2780-2783, 2002.

22. Janne Salo, Johanna Meltaus, Eero Noponen, Martti M Salomaa, Anne Lönnqvist, Tomi Koskinen, Ville Viikari, Jussi Säily, Janne Häkli, Juha Ala-Laurinaho, et al. Holograms for shaping radio-wave fields. Journal of Optics A: Pure and Applied Optics, 4(5):S161, 2002.

23. JL Coutaz, F Garet, E Bonnet, AV Tishchenko, O Parriaux, and M Nazarov. Grating diffraction effects in the THz domain. Acta Phys. Pol. A, 107:26-37, 2005.

24. A Siemion, P Kostrowiecki-Lopata, A Pindur, P Zagrajek, and M Sypek. Paper on designing costless THz paper optics. Advances in Materials Science and Engineering, 2016, 2016.

25. Adolf W Lohmann and S Sinzinger. Optical information processing. Universitätsverlag Ilmenau Ilmenau, 2006.

26. Dong Li, Shiwei Shu, Fangfang Li, Guohong Ma, Ye Dai, and Hongliang Ma. Anomalous transmission of terahertz wave through one-dimensional lamellar metallic grating. Optics Communications, 284(10-11):2415-2419, 2011.

27. Fumiaki Miyamaru and Masanori Hangyo. Anomalous terahertz transmission through double-layer metal hole arrays by coupling of surface plasmon polaritons. Physical Review B, 71(16):165408, 2005.

28. Xiaofeng Li and Siu Fung Yu. Diffraction characteristics of concentric circular metal grating operating at terahertz regime. IEEE Journal of Quantum Electronics, 46(6):898-905, 2010.

29. Zhenwei Xie, Jingwen He, Xinke Wang, Shengfei Feng, and Yan Zhang. Generation of terahertz vector beams with a concentric ring metal grating and photo-generated carriers. Optics letters, 40(3):359-362, 2015.

30. M Nazarov and J-L Coutaz. Terahertz surface waves propagating on metals with sub-wavelength structure and grating reliefs. Journal of Infrared, Millimeter, and Terahertz Waves, 32(10):1054, 2011.

31. John F O'Hara, RD Averitt, and AJ Taylor. Terahertz surface plasmon polariton coupling on metallic gratings. Optics Express, 12(25):6397-6402, 2004.

32. Emilie Hérault, Jean-Louis Coutaz, Agnieszka Siemion, Andrzej Siemion, Michał Makowski, and Maciej Sypek. Prism-like behavior at terahertz frequencies of a $2 \mathrm{~d}$ metallic grid with a varying periodicity. Journal of Infrared, Millimeter, and Terahertz Waves, 32(4):403-406, 2011.

33. Xueqian Zhang, Zhen Tian, Weisheng Yue, Jianqiang Gu, Shuang Zhang, Jiaguang Han, and Weili Zhang. Broadband terahertz wave deflection based on c-shape complex metamaterials with phase discontinuities. Advanced Materials, 25(33):4567-4572, 2013. 
34. Martin S Heimbeck, Patrick J Reardon, John Callahan, and Henry O Everitt. Transmissive quasioptical ronchi phase grating for terahertz frequencies. Optics letters, 35(21):3658-3660, 2010.

35. Ioannis Chatzakis, Philippe Tassin, Liang Luo, Nian-Hai Shen, Lei Zhang, Jigang Wang, Thomas Koschny, and Costas M Soukoulis. One-and two-dimensional photo-imprinted diffraction gratings for manipulating terahertz waves. Applied Physics Letters, 103(4):043101, 2013.

36. AD Squires, Evan Constable, and RA Lewis. 3d printing of aspherical terahertz lenses and diffraction gratings. In Infrared, Millimeter, and Terahertz waves (IRMMW-THz), 2014 39th International Conference on, pages 1-2. IEEE, 2014.

37. AD Squires, E Constable, and RA Lewis. $3 \mathrm{~d}$ printed terahertz diffraction gratings and lenses. Journal of Infrared, Millimeter, and Terahertz Waves, 36(1):72-80, 2015.

38. Stefan F Busch, Marcel Weidenbach, Jan C Balzer, and Martin Koch. THz optics 3d printed with topas. Journal of Infrared, Millimeter, and Terahertz Waves, 37(4):303-307, 2016.

39. Jarosław Suszek, Maciej Sypek, Michał Makowski, Frédéric Garet, Izabela Ducin, Karol Kakarenko, Jarosław Bomba, and Jean-Louis Coutaz. Evaluation of the shadow effect in terahertz kinoform gratings. Optics letters, 38(9):1464-1466, 2013.

40. Benedikt Scherger, Norman Born, Christian Jansen, Steffen Schumann, Martin Koch, and Karin Wiesauer. Compression molded terahertz transmission blaze-grating. IEEE Transactions on Terahertz Science and Technology, 2(5):556-561, 2012.

41. Stefan F Busch, Norman Born, Martin Koch, and Bernd Fischer. Terahertz reflection gratings made by room-temperature high-pressure molding. Journal of Infrared, Millimeter, and Terahertz Waves, 34(7-8):413-415, 2013.

42. Maciej Sypek, Jean-Louis Coutaz, Andrzej Kolodziejczyk, Michal Makowski, and Jaroslaw Suszek. Aberrations of the large aperture attenuating THz lenses. In Terahertz Technology and Applications $V$, volume 8261, page 826110. International Society for Optics and Photonics, 2012.

43. AN Agafonov, BO Volodkin, AK Kaveev, BA Knyazev, GI Kropotov, VS Pavel'ev, VA Soifer, KN Tukmakov, EV Tsygankova, and Yu Yu Choporova. Silicon diffractive optical elements for highpower monochromatic terahertz radiation. Optoelectronics, Instrumentation and Data Processing, 49(2):189-195, 2013.

44. Chia-Jen Lin, Chuan-Hsien Lin, Yu-Tai Li, Ru-Pin Pan, and Ci-Ling Pan. Electrically controlled liquid crystal phase grating for terahertz waves. IEEE Photonics Technology Letters, 21(11):730$732,2009$.

45. James C Wiltse. Diffraction optics for terahertz waves. In Terahertz for Military and Security Applications II, volume 5411, pages 127-136. International Society for Optics and Photonics, 2004.

46. David R Reid and Glenn S Smith. A full electromagnetic analysis of grooved-dielectric fresnel zone plate antennas for microwave and millimeter-wave applications. IEEE transactions on antennas and propagation, 55(8):2138-2146, 2007.

47. James C Wiltse. Stepped conical zone plate antenna. In Photonic and Quantum Technologies for Aerospace Applications III, volume 4386, pages 85-93. International Society for Optics and Photonics, 2001.

48. James C Wiltse. Bandwidth characteristics for the stepped conical-zoned antenna. In Photonic and Quantum Technologies for Aerospace Applications IV, volume 4732, pages 59-69. International Society for Optics and Photonics, 2002.

49. James C Wiltse. Large-angle zone plate antennas. In Enabling Photonic Technologies for Aerospace Applications V, volume 5104, pages 45-57. International Society for Optics and Photonics, 2003.

50. James C Wiltse. Zone plate designs for terahertz frequencies. In Terahertz for Military and Security Applications III, volume 5790, pages 167-180. International Society for Optics and Photonics, 2005.

51. L Leyten and MHAJ Herben. Vectorial far-field analysis of the fresnel-zone plate antenna: A comparison with the parabolic reflector antenna. Microwave and Optical Technology Letters, 5(2):49-56, 1992.

52. LCJ Baggen, CJJ Jeronimus, and MHAJ Herben. The scan performance of the fresnel-zone plate antenna: A comparison with the parabolic reflector antenna. Microwave and Optical Technology Letters, 6(13):769-774, 1993.

53. Hristo D Hristov and Mathieu HAJ Herben. Millimeter-wave fresnel-zone plate lens and antenna. IEEE Transactions on Microwave Theory and Techniques, 43(12):2779-2785, 1995.

54. LP Kamburov, HD Hristov, and R Feick. Optimum design of millimeter-wave double-dielectric fresnel zone-plate lens and antenna. International Journal of Infrared and Millimeter Waves, 25(2):301-316, 2004. 
55. JM Rodriguez, Hristo D Hristov, and Walter Grote. Fresnel zone plate and ordinary lens antennas: Comparative study at microwave and terahertz frequencies. In Microwave Conference (EuMC), 2011 41st European, pages 894-897. IEEE, 2011.

56. Hristo D Hristov. Terahertz harmonic operation of microwave fresnel zone plate lens and antenna: Frequency filtering and space resolution properties. International journal of antennas and propagation, 2011, 2011.

57. HD Hristov, JM Rodriguez, and W Grote. The grooved-dielectric fresnel zone plate: An effective terahertz lens and antenna. Microwave and Optical Technology Letters, 54(6):1343-1348, 2012.

58. Paul F Goldsmith. Zone plate lens antennas for millimeter and submillimeter wavelengths. 1992.

59. Mark A Gouker and Glenn S Smith. A millimeter-wave integrated-circuit antenna based on the fresnel zone plate. IEEE transactions on microwave theory and techniques, 40(5):968-977, 1992.

60. David R Reid and Glenn S Smith. Design of phase-correcting zone plate antennas for microwave and millimeter-wave applications using a full electromagnetic analysis. In Antennas and Propagation Society International Symposium, 2007 IEEE, pages 1505-1508. IEEE, 2007.

61. Edward D Walsby, R Cheung, Richard J Blaikie, and David RS Cumming. Fabrication of multilevel silicon diffractive lenses for terahertz frequencies. In Micromachine Technology for Diffractive and Holographic Optics, volume 3879, pages 79-88. International Society for Optics and Photonics, 1999.

62. ED Walsby, SM Durbin, DRS Cumming, and RJ Blaikie. Analysis of silicon terahertz diffractive optics. Current Applied Physics, 4(2-4):102-105, 2004.

63. ED Walsby, J Alton, CH Worrall, HE Beere, DA Ritchie, J Leach, M Padgett, and DRS Cumming. Fabrication of terahertz holograms. Journal of Vacuum Science \& Technology B: Microelectronics and Nanometer Structures Processing, Measurement, and Phenomena, 25(6):2329-2332, 2007.

64. Shimul C Saha, Chong Li, Yong Ma, James P Grant, and David RS Cumming. Fabrication of multilevel silicon diffractive lens at terahertz frequency. IEEE Transactions on Terahertz Science and Technology, 3(4):479-485, 2013.

65. VS Cherkassky, AV Fanova, LA Merzhievsky, SA Zhigach, Young Uk Jeong, Hyuk Jin Cha, NG Gavrilov, BA Knyazev, GN Kulipanov, IA Polskikh, et al. Diffraction optical elements and optical systems with a high power monochromatic terahertz source. 2007.

66. VM Vedernikov, PM Dutov, AI Kokarev, VP Kir'yanov, BA Knyazev, VG Nikitin, IG Pal'chikova, AR Sametov, MF Stupak, YV Chugui, et al. Diffractive elements for a free electron laser. Optoelectronics, Instrumentation and Data Processing, 46(4):365-375, 2010.

67. AN Agafonov, MG Vlasenko, BO Volodkin, VV Gerasimov, AK Kaveev, BA Knyazev, GI Kropotov, VS Pavelyev, IG Palchikova, VA Soyfer, et al. Diffractive lenses for high-power terahertz radiation beams. Bulletin of the Russian Academy of Sciences: Physics, 77(9):1164-1166, 2013.

68. Maksim Sergeevich Komlenok, Boris Olegovich Volodkin, Boris Aleksandrovich Knyazev, TV Kononenko, VV Kononenko, Vitalii Ivanovich Konov, VA Soifer, VS Pavel'ev, Konstantin Nikolaevich Tukmakov, and Yu Yu Choporova. Fabrication of a multilevel THz fresnel lens by femtosecond laser ablation. Quantum Electronics, 45(10):933, 2015.

69. VS Pavelyev, MS Komlenok, BO Volodkin, BA Knyazev, TV Kononenko, VI Konov, VA Soifer, and $\mathrm{Yu}$ Yu Choporova. Fabrication of high-effective silicon diffractive optics for the terahertz range by femtosecond laser ablation. Physics Procedia, 84:170-174, 2016.

70. BA Knyazev, GN Kulipanov, and NA Vinokurov. Novosibirsk terahertz free electron laser: instrumentation development and experimental achievements. Measurement Science and Technology, 21(5):054017, 2010.

71. Boris A Knyazev, Valery S Cherkassky, Yulia Yu Choporova, Vasily V Gerasimov, Maxim G Vlasenko, Mikhail A Dem'yanenko, and Dmitry G Esaev. Real-time imaging using a high-power monochromatic terahertz source: comparative description of imaging techniques with examples of application. Journal of Infrared, Millimeter, and Terahertz Waves, 32(10):1207-1222, 2011.

72. L Minkevičius, S Indrišiūnas, R Šniaukas, B Voisiat, V Janonis, V Tamošiūnas, I Kašalynas, G Račiukaitis, and G Valušis. Terahertz multilevel phase fresnel lenses fabricated by laser patterning of silicon. Optics letters, 42(10):1875-1878, 2017.

73. Linas Minkevičius, Simonas Indrišiūnas, Ramūnas Šniaukas, Gediminas Račiukaitis, Vytautas Janonis, Vincas Tamošiūnas, Irmantas Kašalynas, and Gintaras Valušis. Compact diffractive optics for THz imaging. Lithuanian Journal of Physics, 58(1), 2018.

74. L Minkevičius, V Tamošiūnas, K Madeikis, B Voisiat, I Kašalynas, and G Valušis. On-chip integration of laser-ablated zone plates for detection enhancement of ingaas bow-tie terahertz detectors. Electronics Letters, 50(19):1367-1369, 2014. 
75. L Minkevicius, B Voisiat, A Mekys, R Venckevicius, I Kasalynas, D Seliuta, G Valusis, G Raciukaitis, and V Tamosiunas. Terahertz zone plates with integrated laser-ablated bandpass filters. Electronics Letters, 49(1):1, 2013.

76. L Minkevičius, K Madeikis, B Voisiat, I Kašalynas, R Venckevičius, G Račiukaitis, V Tamošiūnas, and $\mathrm{G}$ Valušis. Focusing performance of terahertz zone plates with integrated cross-shape apertures. Journal of Infrared, Millimeter, and Terahertz, Waves, 35(9):699-702, 2014.

77. L Minkevičius, K Madeikis, B Voisiat, V Tamosnmas, G Valušis, G Račiukaitis, and I Kašalynas. Compact diffractive optical components for terahertz beam manipulation. In Infrared, Millimeter, and Terahertz waves (IRMMW-THz), 2015 40th International Conference on, pages 1-2. IEEE, 2015.

78. Irmantas Kašalynas, Rimvydas Venckevičius, Linas Minkevičius, Aleksander Sešek, Faustino Wahaia, Vincas Tamošiūnas, Bogdan Voisiat, Dalius Seliuta, Gintaras Valušis, Andrej Švigelj, et al. Spectroscopic terahertz imaging at room temperature employing microbolometer terahertz sensors and its application to the study of carcinoma tissues. Sensors, 16(4):432, 2016.

79. Agnieszka Siemion, Andrzej Siemion, Michał Makowski, Jarosław Suszek, Jarosław Bomba, Adam Czerwiński, Frédéric Garet, Jean-Louis Coutaz, and Maciej Sypek. Diffractive paper lens for terahertz optics. Optics letters, 37(20):4320-4322, 2012.

80. Maciej Sypek, Michał Makowski, Emilie Hérault, Agnieszka Siemion, Andrzej Siemion, Jarosław Suszek, Frédéric Garet, and Jean-Louis Coutaz. Highly efficient broadband double-sided fresnel lens for THz range. Optics letters, 37(12):2214-2216, 2012.

81. Xinke Wang, Zhenwei Xie, Wenfeng Sun, Shengfei Feng, Ye Cui, Jiasheng Ye, and Yan Zhang. Focusing and imaging of a virtual all-optical tunable terahertz fresnel zone plate. Optics letters, 38(22):4731-4734, 2013.

82. Md Itrat Bin Shams, Zhenguo Jiang, Jubaid Qayyum, Syed Rahman, Patrick Fay, and Lei Liu. A terahertz reconfigurable photo-induced fresnel-zone-plate antenna for dynamic two-dimensional beam steering and forming. In Microwave Symposium (IMS), 2015 IEEE MTT-S International, pages 1-4. IEEE, 2015.

83. Md Itrat B Shams, Zhenguo Jiang, Syed M Rahman, Li-Jing Cheng, Jeffrey L Hesler, Patrick Fay, and Lei Liu. A 740-ghz dynamic two-dimensional beam-steering and forming antenna based on photo-induced reconfigurable fresnel zone plates. IEEE Transactions on Terahertz Science and Technology, 7(3):310-319, 2017.

84. Feng Hui and Wang Li. A zone plate as a tunable terahertz filter. Chinese Physics Letters, 27(6):064201, 2010.

85. Alicia E Torres-Garcia, Bakhtiyar Orazbayev, Inigo Ederra, and Ramon Gonzalo. Ir-fresnel zone plate lens acting as THz antenna. In Millimeter-Waves, 2017 10th Global Symposium on, pages 129-131. IEEE, 2017.

86. Z Zhang and W Dou. Binary diffractive small lens array for THz imaging system. Journal of Electromagnetic Waves and Applications, 25(2-3):177-187, 2011.

87. Xuecou Tu, Lin Kang, Chao Wan, Lei Xu, Qingkai Mao, Peng Xiao, Xiaoqing Jia, Wenbin Dou, Jian Chen, and Peiheng Wu. Diffractive microlens integrated into nb $5 \mathrm{n} 6$ microbolometers for $\mathrm{THz}$ detection. Optics express, 23(11):13794-13803, 2015.

88. Krzesimir Szkudlarek, Maciej Sypek, Grzegorz Cywiński, Jarosław Suszek, Przemysław Zagrajek, Anna Feduniewicz-Żmuda, Ivan Yahniuk, Sergey Yatsunenko, Anna Nowakowska-Siwińska, Dominique Coquillat, et al. Terahertz $3 \mathrm{~d}$ printed diffractive lens matrices for field-effect transistor detector focal plane arrays. Optics express, 24(18):20119-20131, 2016.

89. Qiu Wang, Xueqian Zhang, Yuehong Xu, Zhen Tian, Jianqiang Gu, Weisheng Yue, Shuang Zhang, Jiaguang Han, and Weili Zhang. A broadband metasurface-based terahertz flat-lens array. Advanced Optical Materials, 3(6):779-785, 2015.

90. IV Minin and OV Minin. Advantages in THz diffractive optical elements. In Infrared and Millimeter Waves, 2004 and 12th International Conference on Terahertz Electronics, 2004. Conference Digest of the 2004 Joint 29th International Conference on, pages 371-372. IEEE.

91. IV Minin and OV Minin. Scanning properties of the diffractive lens-plus-axicon lens in THz. In Proceedings of the 11th Microcoll Symposium, pages 233-236, 2003.

92. GA Turnbull, DA Robertson, GM Smith, L Allen, and MJ Padgett. The generation of free-space laguerre-gaussian modes at millimetre-wave frequencies by use of a spiral phaseplate. Optics communications, 127(4-6):183-188, 1996.

93. Agnieszka Siemion, Andrzej Siemion, Michał Makowski, Maciej Sypek, Emilie Hérault, Frédéric Garet, and Jean-Louis Coutaz. Off-axis metallic diffractive lens for terahertz beams. Optics letters, 36(11):1960-1962, 2011. 
94. Walter D Furlan, Vicente Ferrando, Juan A Monsoriu, Przemysław Zagrajek, Elżbieta Czerwińska, and Mieczysław Szustakowski. 3d printed diffractive terahertz lenses. Optics letters, 41(8):1748$1751,2016$.

95. D Jokubauskis, L Minkevičius, M Karaliūnas, S Indrišiūnas, I Kašalynas, G Račiukaitis, and G Valušis. Fibonacci terahertz imaging by silicon diffractive optics. Optics letters, 43(12):2795-2798, 2018.

96. Jaroslaw Suszek, Agnieszka Siemion, Marcin S Bieda, Narcyz Błocki, Dominique Coquillat, Grzegorz Cywiński, Elżbieta Czerwińska, Marta Doch, Adam Kowalczyk, Norbert Palka, Artur Sobczyk, Przemysław Zagrajek, Marcin Zaremba, Andrzej Kolodziejczyk, Wojciech Knap, and Maciej Sypek. 3-d-printed flat optics for $\mathrm{THz}$ linear scanners. IEEE transactions on Terahertz Science and Technology, 5(2):314-316, 2015.

97. Agnieszka Siemion, Andrzej Siemion, Jaroslaw Suszek, Adam Kowalczyk, Jaroslaw Bomba, Artur Sobczyk, Norbert Palka, Przemysław Zagrajek, Andrzej Kolodziejczyk, and Maciej Sypek. THz beam shaping based on paper diffractive optics. IEEE Transactions on Terahertz Science and Technology, 6(4):568-575, 2016.

98. Karolina Liebert, M Rachon, Agnieszka Siemion, Jarosław Suszek, D But, W Knap, and Maciej Sypek. THz beam shaper realizing fan-out patterns. Journal of Infrared, Millimeter, and Terahertz. Waves, 38(8):1019-1030, 2017.

99. Y Liu, H Shi, J Ma, D Gan, J Cui, C Du, and X Luo. Directional terahertz radiation through grooveassisted hole array. Applied Physics B, 92(4):623, 2008.

100. M Rachon, K Liebert, A Siemion, J Bomba, A Sobczyk, Wojciech Knap, Dominique Coquillat, J Suszek, and M Sypek. Geometrical aberration suppression for large aperture sub-THz lenses. Journal of Infrared, Millimeter, and Terahertz Waves, 38(3):347-355, 2017.

101. Changming Liu, Liting Niu, Kejia Wang, and Jinsong Liu. 3d-printed diffractive elements induced accelerating terahertz airy beam. Optics express, 24(25):29342-29348, 2016.

102. Igor Minin and Oleg Minin. Active mmw/terahertz security system based on bessel beams. ISRN Optics, 2013, 2013.

103. VS Pavelyev, BO Volodkin, KN Tukmakov, BA Knyazev, and Yu Yu Choporova. Transmissive diffractive microoptics for high-power THz laser radiation. In AIP Conference Proceedings, volume 1989, page 020025. AIP Publishing, 2018.

104. Yanzhong Yu and Wenbin Dou. Generation of pseudo-bessel beams at $\mathrm{THz}$ frequencies by use of binary axicons. Optics express, 17(2):888-893, 2009.

105. Xuli Wei, Changming Liu, Liting Niu, Zhongqi Zhang, Kejia Wang, Zhengang Yang, and Jinsong Liu. Generation of arbitrary order bessel beams via $3 \mathrm{~d}$ printed axicons at the terahertz frequency range. Applied optics, 54(36):10641-10649, 2015.

106. K Miyamoto, K Suizu, T Akiba, and T Omatsu. Direct observation of the topological charge of a terahertz vortex beam generated by a tsurupica spiral phase plate. Applied Physics Letters, 104(26):261104, 2014.

107. BA Knyazev, Yu Yu Choporova, MS Mitkov, VS Pavelyev, and BO Volodkin. Generation of terahertz surface plasmon polaritons using nondiffractive bessel beams with orbital angular momentum. Physical review letters, 115(16):163901, 2015.

108. Changming Liu, Xuli Wei, Liting Niu, Kejia Wang, Zhengang Yang, and Jinsong Liu. Discrimination of orbital angular momentum modes of the terahertz vortex beam using a diffractive mode transformer. Optics express, 24(12):12534-12541, 2016.

109. Jingwen He, Xinke Wang, Dan Hu, Jiasheng Ye, Shengfei Feng, Qiang Kan, and Yan Zhang. Generation and evolution of the terahertz vortex beam. Optics express, 21(17):20230-20239, 2013.

110. Jingwen He, Sen Wang, and Yan Zhang. Terahertz beam shaping with metasurface. In Infrared, Millimeter-Wave, and Terahertz. Technologies IV, volume 10030, page 1003019. International Society for Optics and Photonics, 2016.

111. Zhenwei Xie, Xinke Wang, Jiasheng Ye, Shengfei Feng, Wenfeng Sun, Tahsin Akalin, and Yan Zhang. Spatial terahertz modulator. Scientific Reports, 3:3347, 2013.

112. AI Hernandez-Serrano, M Weidenbach, SF Busch, M Koch, and E Castro-Camus. Fabrication of gradient-refractive-index lenses for terahertz applications by three-dimensional printing. JOSA $B$, 33(5):928-931, 2016.

113. Takanori Okada and Koichiro Tanaka. Photo-designed terahertz devices. Scientific reports, 1:121, 2011.

114. Pai-Yen Chen and Andrea Alù. THz beamforming using graphene-based devices. In Radio and Wireless Symposium (RWS), 2013 IEEE, pages 55-57. IEEE, 2013. 
115. Qi-Ye Wen, Wei Tian, Qi Mao, Zhi Chen, Wei-Wei Liu, Qing-Hui Yang, Matthew Sanderson, and Huai-Wu Zhang. Graphene based all-optical spatial terahertz modulator. Scientific reports, 4:7409, 2014.

116. Wai Lam Chan, Hou-Tong Chen, Antoinette J Taylor, Igal Brener, Michael J Cich, and Daniel M Mittleman. A spatial light modulator for terahertz beams. Applied Physics Letters, 94(21):213511, 2009.

117. Sergei A Kuznetsov, Mikhail A Astafev, Miguel Beruete, and Miguel Navarro-Cía. Planar holographic metasurfaces for terahertz focusing. Scientific reports, 5:7738, 2015.

118. Ralph W Gerchberg. A practical algorithm for the determination of phase from image and diffraction plane pictures. Optik, 35:237-246, 1972.

119. Jan Gospodaric, Artem Kuzmenko, Anna Pimenov, Christian Huber, Dieter Suess, Stefan Rotter, and Andrei Pimenov. 3d-printed phase waveplates for THz beam shaping. Applied Physics Letters, 112(22):221104, 2018.

120. M Nazarov and J-L Coutaz. Terahertz surface waves propagating on metals with sub-wavelength structure and grating reliefs. Journal of Infrared, Millimeter, and Terahertz Waves, 32(10):1054, 2011.

121. Yinghao Yuan, Jinsong Liu, Jian He, and Jianquan Yao. Directional terahertz beams realized by depth-modulated metallic surface grating structures. JOSA B, 28(11):2674-2679, 2011.

122. Christian Nadell, Kebin Fan, and Willie Padilla. Resonance-domain diffractive lens for the terahertz region. Optics letters, 43(10):2384-2387, 2018.

123. Igor V Minin, Oleg V Minin, Yevgeny R Triandaphilov, and Victor V Kotlyar. Subwavelength diffractive photonic crystal lens. Progress In Electromagnetics Research, 7:257-264, 2008.

124. Kazuo Shiraishi, Satoshi Oyama, and Chen S Tsai. A polarizer using thin metallic-film subwavelength grating for infrared to terahertz region. Journal of Lightwave Technology, 29(5):670-676, 2011.

125. Shin-ichi Kuroo, Satoshi Oyama, Kazuo Shiraishi, Hiroyuki Sasho, and Kazushiro Fukushima. Reduction of light reflection at silicon-plate surfaces by means of subwavelength gratings in terahertz region. Applied optics, 49(15):2806-2812, 2010.

126. Jarosław Bomba, Jarosław Suszek, Michał Makowski, Artur Sobczyk, and Maciej Sypek. 3-d printed anti-reflection structures for the terahertz region. Journal of Infrared, Millimeter, and Terahertz Waves, 39(1):24-35, 2018.

127. Milda Tamosiunaite, Simonas Indrisiunas, Vincas Tamosiunas, Linas Minkevicius, Andrzej Urbanowicz, Gediminas Raciukaitis, Irmantas Kasalynas, and Gintaras Valusis. Focusing of terahertz radiation with laser-ablated antireflective structures. IEEE Transactions on Terahertz Science and Technology, 2018.

128. Xiao-Yan Jiang, Jia-Sheng Ye, Jing-Wen He, Xin-Ke Wang, Dan Hu, Sheng-Fei Feng, Qiang Kan, and Yan Zhang. An ultrathin terahertz lens with axial long focal depth based on metasurfaces. Optics Express, 21(24):30030-30038, 2013.

129. Ruth Vilar Mateo, Romain Czarny, Mane-Si Laure Lee, Brigitte Loiseaux, Maciej Sypek, Michal Makowski, Cedric Martel, Thomas Crepin, Fabrice Boust, Ronald Joseph, et al. Q-band millimeterwave antennas: An enabling technology for multigigabit wireless backhaul. In IEEE Microwave Magazine, volume 15, pages 121-130. Institute of Electrical and Electronics Engineers (IEEE), 2014.

130. Ruth Vilar Mateo, Javier Martí Sendra, R Czarny, M Sypek, M Makowski, C Martel, T Crépin, F Boust, R Joseph, K Herbertz, et al. Smart antennas and front-end modules in q-band for backhaul networks. In Microwave Journal, pages 28-34. Horizon House Publications, Inc., 2014.

131. Jingwen He, Jiasheng Ye, Xinke Wang, Qiang Kan, and Yan Zhang. A broadband terahertz ultrathin multi-focus lens. Scientific reports, 6:28800, 2016.

132. Dan Hu, Xinke Wang, Shengfei Feng, Jiasheng Ye, Wenfeng Sun, Qiang Kan, Peter J Klar, and Yan Zhang. Ultrathin terahertz planar elements. Advanced Optical Materials, 1(2):186-191, 2013.

133. Quanlong Yang, Jianqiang Gu, Dongyang Wang, Xueqian Zhang, Zhen Tian, Chunmei Ouyang, Ranjan Singh, Jiaguang Han, and Weili Zhang. Efficient flat metasurface lens for terahertz imaging. Optics Express, 22(21):25931-25939, 2014.

134. Chun-Chieh Chang, Daniel Headland, Derek Abbott, Withawat Withayachumnankul, and Hou-Tong Chen. Demonstration of a highly efficient terahertz flat lens employing tri-layer metasurfaces. Optics letters, 42(9):1867-1870, 2017. 
135. Daniel Headland, Chun-Chieh Chang, Derek Abbott, Withawat Withayachumnankul, and Hou-Tong Chen. Efficient terahertz metasurface-based flat lens. In Infrared, Millimeter, and Terahertz Waves (IRMMW-THz), 2017 42nd International Conference on, pages 1-2. IEEE, 2017.

136. Huan Yi, Shi-Wei Qu, Bao-Jie Chen, Xue Bai, Kung Bo Ng, and Chi Hou Chan. Flat terahertz reflective focusing metasurface with scanning ability. Scientific Reports, 7(1):3478, 2017.

137. Hailong Zhou, Jianji Dong, Siqi Yan, Yifeng Zhou, and Xinliang Zhang. Generation of terahertz vortices using metasurface with circular slits. IEEE Photonics Journal, 6(6):1-7, 2014.

138. Masayoshi Tonouchi. Cutting-edge terahertz technology. Nature photonics, 1(2):97, 2007.

139. Kodo Kawase, Yuichi Ogawa, Yuuki Watanabe, and Hiroyuki Inoue. Non-destructive terahertz imaging of illicit drugs using spectral fingerprints. Optics express, 11(20):2549-2554, 2003.

140. JB Jackson, M Mourou, JF Whitaker, IN Duling III, SL Williamson, M Menu, and GA Mourou. Terahertz imaging for non-destructive evaluation of mural paintings. Optics communications, 281(4):527-532, 2008.

141. I Amenabar, F Lopez, and A Mendikute. In introductory review to THz non-destructive testing of composite mater. Journal of Infrared, Millimeter, and Terahertz Waves, 34(2):152-169, 2013.

142. John F Federici, Brian Schulkin, Feng Huang, Dale Gary, Robert Barat, Filipe Oliveira, and David Zimdars. THz imaging and sensing for security applicationsexplosives, weapons and drugs. Semiconductor Science and Technology, 20(7):S266, 2005.

143. Demian Tinkiel, David White, and Neil A Trappe. Efficient design of THz security systems. In Security Technology (ICCST), 2011 IEEE International Carnahan Conference on, pages 1-9. IEEE, 2011.

144. Norbert Palka. Identification of concealed materials, including explosives, by terahertz reflection spectroscopy. Optical Engineering, 53(3):031202, 2013.

145. Ian S Gregory, Robert K May, Ke Su, and J Axel Zeitler. Terahertz car paint thickness sensor: Out of the lab and into the factory. In Infrared, Millimeter, and Terahertz waves (IRMMW-THz), 2014 39th International Conference on, pages 1-1. IEEE, 2014.

146. Soufiene Krimi, Jens Klier, Joachim Jonuscheit, Georg von Freymann, Ralph Urbansky, and René Beigang. Highly accurate thickness measurement of multi-layered automotive paints using terahertz technology. Applied Physics Letters, 109(2):021105, 2016.

147. Ke Su, Yao-Chun Shen, and J Axel Zeitler. Terahertz sensor for non-contact thickness and quality measurement of automobile paints of varying complexity. IEEE Transactions on Terahertz Science and Technology, 4(4):432-439, 2014.

Publisher's Note Springer Nature remains neutral with regard to jurisdictional claims in published maps and institutional affiliations. 